\title{
Fetal magnetocardiographic mapping using independent component analysis
}

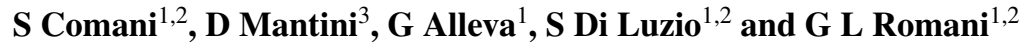 \\ ${ }^{1}$ Department of Clinical Sciences and Bio-imaging, Chieti University, Italy \\ 2 ITAB-Institute of Advanced Biomedical Technologies, University Foundation 'G D'Annunzio', \\ Chieti University, Italy \\ ${ }^{3}$ Department of Informatics and Automation Engineering, Marche Polytechnic University, \\ Ancona, Italy \\ E-mail: comani@itab.unich.it
}

Received 25 June 2004, accepted for publication 22 September 2004

Published 13 October 2004

Online at stacks.iop.org/PM/25/1459

doi:10.1088/0967-3334/25/6/011

\begin{abstract}
Fetal magnetocardiography (fMCG) is the only noninvasive technique allowing effective assessment of fetal cardiac electrical activity during the prenatal period. The reconstruction of reliable magnetic field mapping associated with fetal heart activity would allow three-dimensional source localization. The efficiency of independent component analysis (ICA) in restoring reliable fetal traces from multichannel fMCG has already been demonstrated. In this paper, we describe a method of reconstructing a complete set of fetal signals hidden in multichannel fMCG preserving their correct spatial distribution, waveform, polarity and amplitude. Fetal independent components, retrieved with an ICA algorithm (FastICA), were interpolated (fICI method) using information gathered during FastICA iterations. The restored fetal signals were used to reconstruct accurate magnetic mapping for every millisecond during the average beat. The procedure was validated on $\mathrm{AMCG}$ recorded from the 22nd gestational week onward with a multichannel MCG system working in a shielded room. The interpolated traces were compared with those obtained with a standard technique, and the consistency of fetal mapping was checked evaluating source localizations relative to fetal echocardiographic information. Good magnetic field distributions during the P-QRS-T waves were attained with fICI for all gestational periods; their reliability was confirmed by threedimensional source localizations.
\end{abstract}

Keywords: fetal magnetocardiography (fMCG), independent component analysis (ICA), signal processing, fetal magnetocardiographic mapping, prenatal diagnosis 


\section{Introduction}

Magnetocardiography (MCG) is a noninvasive technique that monitors the spontaneous activity of the heart by measuring the weak magnetic field variations associated with the primary ionic currents flowing through the myocardium during the cardiac cycle (Siltanen 1989). Since superconducting multi-channel systems have become available, the cardiac magnetic field can be recorded with great sensitivity simultaneously from a number of positions over the chest (Pasquarelli and Di Luzio1993, Tavarozzi et al 2002a), hence allowing accurate field mapping during the cardiac cycle, useful for several clinical applications, particularly for arrhythmias (Weismuller et al 1992, Brockmeier et al 1997, Muller et al 1999, Hren et al 1999, Kandori et al 2001, Comani et al 2001, 2003, 2004a, Tavarozzi et al 2002b).

Fetal magnetocardiography (fMCG) is the application of MCG to the study of fetal heart function and it records the mixed maternal and fetal cardiac signals above the maternal abdomen. Depending on the quality of fMCG traces and on the effectiveness of the technique used, fetal signals, generally one order of magnitude smaller than the maternal ones, can be retrieved and used for the prenatal classification of fetal cardiac dysfunctions, mainly during the second half of pregnancy when the insulating properties of vernix caseosa strongly reduce the quality of trans-abdominal fetal ECG, while leaving fMCG almost unaffected (Wakai et al 2000a, 2000b, 2003, Menendez et al 2000, Khaler et al 2001, Horigome et al 2001, Peters et al 2001, Hosono et al 2002, Lewis 2003, Comani et al 2004b).

Given the large fetal heart rate variability, rhythm characterization performed in time, frequency and space would be extremely welcome. Consistent analysis in time and/or frequency can be performed on few reliable fetal traces or on averaged beats (Kotini et al 2001, Kaler et al 2002, Stinstra et al 2002, Grimm et al 2003, Lowery et al 2003, Comani et al 2004c), while dependable field mapping throughout the cardiac cycle, although useful to detect early signs of ventricular repolarization instability and/or to achieve precise localization of arrhythmogenic foci and associated accessory pathways (Muller et al 1999, Comani et al 2003, Wakai et al 2000b, 2003, Menendez et al 2001), might not be available with the required precision, which in fetal studies is even more important, given the small dimensions of the fetal heart (St John Sutton et al 1984).

The accuracy of inverse source localizations strongly depends on the total number of traces available to reconstruct field maps and on their signal-to-noise ratio (SNR). By means of an independent component analysis algorithm (FastICA), the authors have achieved high quality reconstructions of fetal signals allowing detailed and reliable cardiac time interval evaluations and arrhythmia characterization in time and frequency domains (Comani et al 2004b, 2004c, 2004d, 2004e). However, the use of FastICA, which is based on neural networks, did not maintain a detailed field mapping because it permitted the retrieval of few fetal signals from many real-time fMCG recordings (Comani et al 2004d, 2004e, Mantini et al 2004). In this paper we present a method based on the interpolation of fetal independent components (fICI method) retrieved with FastICA. This procedure demonstrated its ability in restoring all fetal signals included in multichannel fMCG preserving their correct space distribution and recovering the original signal waveform, polarity and amplitude. Fetal isofield maps were reconstructed from fMCG recorded at different gestational age, and for each data set the fetal traces interpolated with fICI were compared with those retrieved with a standard deterministic technique; field mapping consistency was estimated with respect to cardiac source localizations, which were compared with fetal echocardiographic information, whenever available. 


\section{Materials and methods}

\subsection{MCG system and fetal recordings}

fMCG recordings were acquired with a 77-channel system; each channel consisted of low-temperature dc-superconducting quantum interference device (dc-SQUID) integrated magnetometer able to measure magnetic field variations ranging from $0.01 \mathrm{pT}$ to $100 \mathrm{pT}$ and to sense overall white noise density of about $5 \mathrm{fT} \mathrm{Hz}^{-\frac{1}{2}}$ above $60 \mathrm{~Hz}$. All channels were kept at $4 \mathrm{~K}$ inside a cylindrical cryostat; the 55 sensing magnetometers were homogeneously distributed on a circular area of $230 \mathrm{~mm}$ diameter (sensing plane) at fixed sensor-to-sensor distances of $32 \mathrm{~mm}$ and at $18 \mathrm{~mm}$ from the cryostat bottom. The remaining magnetometers, positioned at higher planes, could be used for residual noise reduction (Della Penna et al 2000).

fMCG signals were acquired from 32 singleton normal pregnancies (maternal age 2138 years); the study protocol has been approved by the Ethics Committee of our University and all volunteers, enrolled during a routine M-2D pulsed color Doppler echocardiographic evaluation of the fetal heart, gave their written informed consent.

fMCG exams were performed from the 22 nd to the 37 th gestational week for a total of $60 \mathrm{fMCG}$ data sets. Each acquisition, performed in a magnetically shielded room, lasted 10 min and provided a set of 55 simultaneous fMCG recordings; sampling frequency was $1 \mathrm{kHz}$ after decimation; data were filtered between $0.016 \mathrm{~Hz}$ and $250 \mathrm{~Hz}$. Maternal ECG was acquired with three amagnetic leads during fMCG.

\subsection{ICA and fetal signal recovering}

ICA is a digital signal processing (DSP) technique able to separate the independent components of mixed signals produced by distinct sources. When applied to fMCG, this basic ICA hypothesis was satisfied because the maternal and fetal hearts are physically separate sources that generate independent cardiac signals overlapped in fMCG.

ICA effectiveness in using fMCG recordings to reconstruct dependable fetal signals, which means stable and reproducible signals with high SNR and clear morphology so that cardiac waves can be detected on single cycles, has already been demonstrated, as well as ICA superior performances with respect to a classical deterministic technique (Comani et al 2004d, 2004e). Consequently, the procedure used to retrieve fetal traces from fMCG will only be shortly outlined.

Given a set of $n \mathrm{fMCG}$ recordings $\left[x_{1}(t), x_{2}(t), \ldots, x_{n}(t)\right]$, where $t$ is time, the ICA model assumes that the $n$ recordings $x_{j}(t)$ are generated by a mixture of $m$ independent components $\left[s_{1}(t), s_{2}(t), \ldots, s_{m}(t)\right]$ with the following linear expansion:

$$
x(t)=A s(t)
$$

where $A$ is a constant full-rank $[n \times m]$ matrix called the mixing matrix. The solution consists in estimating $A$ and $s(t)$ from $x(t)$ under the condition that $s_{i}(t)$ must be as independent as possible, for $i=1, \ldots, m$.

The ICA problem can be solved under the condition that the number of independent components $(m)$ is always smaller than or equal to the number of mixtures $(n)$; in order to obtain one reliable fetal signal a large number of input traces had to be used, hence losing the detailed spatial information on the fetal cardiomagnetic field provided with the 55 sensing channels. 
2.2.1. $f M C G$ pre-processing. In order to maximize the number of recovered fetal traces, fMCG recordings were centered by subtracting the $j$ th average value from each $x_{j}(t)$, so that the vector $s(t)$ became zero-mean (Hyvärinen 1999). Whitening was then performed with eigenvalue decomposition (EVD) of the covariance matrix (Yang and Wang 1999), linearly transforming a vector $x(t)$ into another vector $\bar{x}(t)$ whose components were uncorrelated and with variances equal to unity. Finally, fMCG were filtered with a second-order Chebyshev filter, band-pass $0.4-150.0 \mathrm{~Hz}$ (Stinstra et al 2002), and with a notch filter at $50 \mathrm{~Hz}$.

2.2.2. Application of FastICA to fMCG. The fixed-point algorithm (FastICA) was chosen to separate fetal and maternal signals because of its efficiency and reliability in achieving convergence (Hyvärinen and Oja 2000). The mutual statistical independence of signal components characterized by probability distributions other than Gaussian, except noise, was assessed minimizing negentropy (Hyvärinen 1999). The components containing fetal information were selected on the basis of heart rate (always higher for the fetus in an approximate ratio of $3: 2$ with respect to the mother) and merged with a weighted algorithm to restore a complete fetal signal.

The fetal signals being extremely weak, sometimes less than background noise, SNR in reconstructed fetal traces could be improved using a smoothing procedure based on a Kaiser filter working with a moving window (Mitra and Kaiser 1993). The smoothing factor $\sigma$, defined as the number of samples in the window, was set inversely proportional to the signal amplitude: approximate values of 12 and $50 \mathrm{~ms}$ were used respectively for rapid and slow cardiac occurrences. The time resolution of retrieved fetal signals was preserved because the window was shifted of $1 \mathrm{~ms}$ at each iteration, and signal intensity at a given instant was obtained averaging all 'smoothed' amplitudes (Comani et al 2004d).

\subsection{Fetal MCG mapping reconstruction}

Henceforward we will describe the procedure used to recover a complete set of 55 fetal signals interpolating the fetal traces retrieved with FastICA (fICI method).

2.3.1. Channel clustering. As described in previous papers (Comani et al 2004e, Mantini et al 2004), a clustering technique was tailored to our MCG system in order to maximize the number of reliable fetal traces that could be obtained from 55 simultaneous fMCG recordings. We further improved this procedure and succeeded in retrieving one fetal signal, having the required quality and dependability, from a cluster of 19 fMCG recordings. The 55 channels were therefore grouped in nine clusters $C_{i}$, partially overlapping and homogeneously distributed over the sensing plane; one cluster was in the middle of the recording area, while the others were peripheral and rotated of $45^{\circ}$ with respect to the adjacent ones. Therefore, nine fetal signals $r_{i}(t)$, uniformly allocated on the sensing plane, were restored with nine FastICA iterations using all clusters.

2.3.2. Interpolation matrix and comprehensive fetal signals retrieval. The implemented interpolation procedure employed information contained in mixing matrix $A$, which contains coefficients that quantify the relative contribution of each retrieved fetal component to each fMCG recording. After all FastICA iterations, nine mixing matrices $A_{i}$ were available. During fetal signal reconstruction, nine vectors $B_{i}$ of 19 elements each were gathered summing, element-by-element, the $A_{i}$ columns containing fetal components. Each $b_{i}$ element measured the contribution of the $i$ th retrieved fetal signal to the $j$ th fMCG recording. 
In general, 55 fetal signals $f_{j}(t)$, which were mixed with maternal components and noise in fMCG recordings, could be restored from nine fetal traces $r_{i}(t)$; this relation can be expressed in algebraic form as

$$
f_{j}(t)=\sum_{i=1}^{9} n_{j i} r_{i}(t)
$$

where $n_{j i}$ are the elements of the [55 $\times 9$ ] interpolation matrix $N$, the columns of which were reconstructed from $B_{i}$. In fact, the relative contribution of the $i$ th fetal trace $r_{i}(t)$ to the $j$ th fetal signal $f_{j}(t)$ depended on whether the $j$ th fMCG recording belonged to cluster $C_{i}$ : in this case the element $n_{j i}$ was set equal to the corresponding element of vector $B_{i}$, otherwise it was set equal to zero. This procedure allowed retrieving all fetal signals with the original spatial distribution.

2.3.3. Amplitude and polarity restoring of averaged fetal beats. The correct amplitudes and polarities of $f_{j}(t)$ were recovered using raw fMCG recordings. The $t_{k}$ time instants corresponding to R peaks, for $k=1, \ldots, N_{\text {beats }}$, were identified and a subset of cycles exhibiting similar duration at $97 \%$ confidence level was selected from each fetal signal and used to compute the average beat $F_{j}(\tau)$ triggering on R peaks. Pre- and post-trigger time values were used to correctly reconstruct $P$ and $T$ waves: $D$ being equal to the average RR duration, $D_{\text {pre }}$ and $D_{\text {post }}$ were set equal to $5 D / 12$ and $7 D / 12$.

Peak-to-peak QRS amplitude $b_{j}$ of $F_{j}(\tau)$ was then calculated as

$$
b_{j}=F_{j}(0)-F_{j}\left(\tau^{\prime}\right)
$$

where $F_{j}(0)$ was the signal intensity at $\mathrm{R}$ peak and $F_{j}\left(\tau^{\prime}\right)$ was the minimum signal value during QRS complex.

The average fetal beat $X_{j}(\tau)$ of the $j$ th fMCG recording and its peak-to-peak QRS amplitude $c_{j}$ were determined with a similar procedure.

Finally, the averaged fetal beat $F_{j}(\tau)$ was scaled according to

$$
F_{j}^{\prime}(\tau)=F_{j}(\tau) \frac{c_{j}}{b_{j}}
$$

Moreover, since coefficients $c_{j}$ and $b_{j}$ might be either positive or negative, trace polarity was also appropriately restored; $c_{j}$ and $b_{j}$ might be used to re-establish the correct amplitude and polarity of $f_{j}(t)$ signals as well.

2.3.4. Cardiac isofield mapping. Magnetic isofield maps associated with fetal cardiac activity could be reconstructed from $F_{j}^{\prime}(\tau)$ every millisecond with a sampling frequency of $1 \mathrm{kHz}$. After the selection of the time instant $\tau_{m}$ for which an isofield map was required, a 55-element vector containing all $F_{j}^{\prime}\left(\tau_{m}\right)$ values was assembled; each $F_{j}^{\prime}\left(\tau_{m}\right)$ element had spatial coordinates corresponding to the location of the $j$ th MCG sensor. The fetal isofield map at instant $\tau_{m}$ was drawn from that allotment. Using a series of consecutive maps, a bi-dimensional description of fetal cardiac activity during the average cycle was available for analysis.

\subsection{Validation of fetal magnetic mapping}

2.4.1. Reliability of reconstructed fetal signals. Fetal traces $f_{j}(t)$, reconstructed and scaled with fICI, were compared with traces $g_{j}(t)$ obtained by means of a deterministic technique that subtracts the averaged maternal beat from fMCG recordings with an adaptive moving window (AMBS) (Comani et al 2003, 2004d). Waveform similarity was estimated on average 
beats calculating the coupled correlation coefficients $c c_{j}$ between sets of $F_{j}^{\prime}(t)$ and $G_{j}(t)$, for $j=1, \ldots, 55$. The correspondence of signal intensities was evaluated on QRS peak-topeak amplitudes for $F_{j}^{\prime}(t)$ and $G_{j}(t)$ pairs, for $j=1, \ldots, 55$. The average amplitude and the minimum and maximum values of background noise in raw fMCG recordings were also provided for comparison.

2.4.2. Effectiveness of reconstructed field mapping for localization purposes. The reliability of fetal isofield mapping obtained with fICI was tested verifying the accuracy of electrical source localizations for P-QRS-T waves. The space distribution of sources generating a magnetic field distribution is not unique, and the identification of cardiac activation sources from $\mathrm{fMCG}$ requires assumptions on the charge distribution in the fetal heart during a cycle and on the characteristics of the surrounding medium. Because of the small dimensions of the fetal heart, the equivalent current dipole (ECD) model and the homogeneous half-space are suitable to represent, in first approximation, fetal myocardial activation throughout the cardiac cycle. ECD is generally appropriate to depict the activation of small structures, such as the atrioventricular node, which corresponds to the onset of ventricular depolarization and is easily localized on echocardiography. The comparison between source localizations obtained with fICI and AMBS was therefore performed for the beginning of R wave; although the chosen models might not be ideal to describe atrial depolarization and ventricular repolarization, they were nonetheless used for source localization during $\mathrm{P}$ and $\mathrm{T}$ waves with the aim of verifying the correctness of relative source positioning.

Inverse localization of activation source position $(x, y, z)$ was first performed with respect to the MCG reference system, then converted into a maternal coordinate system having the origin in the maternal abdomen projection of the fetal heart center. This transformation of coordinates was necessary to compare source localizations with ultrasonographic information, always provided with respect to the surface of the maternal abdomen. Source localization was then given as

$$
\left\{\begin{array}{l}
x^{\prime}=x-d_{1} \cos \varphi \cos \theta \\
y^{\prime}=y-d_{1} \cos \varphi \sin \theta \\
z^{\prime}=z+d_{2}-d_{1} \sin \varphi
\end{array}\right.
$$

where $d_{1}=7 \mathrm{~mm}$ was the average distance between the maternal abdomen and the cryostat bottom, and $d_{2}=18 \mathrm{~mm}$ was the distance between the cryostat bottom and the sensing plane. Angles $\varphi$ and $\theta$ were defined as

$$
\left\{\begin{array}{l}
\theta=\arctan \left(\frac{y}{x}\right) \\
\varphi=\arctan \left(\frac{z+d_{2}}{\sqrt{x^{2}+y^{2}}}\right) .
\end{array}\right.
$$

For each fetus, heart position and depth were measured, while its dimensions were calculated from the echocardiographic measure of: the distance between maternal abdomen and fetal heart center $\left(b_{0}\right)$, fetal heart length $\left(b_{1}\right)$ and fetal heart width at interception between atria and ventricula $\left(b_{2}\right)$. The atrio-ventricular node position was then given by

$$
\left\{\begin{array}{l}
x_{E}=b_{0} \cos \varphi \cos \theta \\
y_{E}=b_{0} \cos \varphi \sin \theta \\
z_{E}=b_{0} \sin \varphi .
\end{array}\right.
$$

Approximate tolerance limits maximizing the acceptable distance between the positions of sources and the atrio-ventricular node were determined for $\mathrm{P}, \mathrm{R}$ and $\mathrm{T}$ waves as

$$
T_{\mathrm{P}}=\frac{b_{1}}{3} \quad T_{\mathrm{R}}=\frac{b_{2}}{3} \quad T_{\mathrm{T}}=\frac{b_{1}}{2} .
$$


Table 1. Comparison between fetal signals restored with fICI and AMBS. Figures are average values referring to fMCG performed during the same gestational period.

\begin{tabular}{lllll}
\hline Gestational period (weeks) & & $22-27$ & $28-32$ & $33-37$ \\
\hline Number of available fMCG recording sets & & 18 & 19 & 23 \\
Number of sets of 55 & fICI & 11 & 17 & 23 \\
reconstructed fetal traces & AMBS & 1 & 12 & 19 \\
QRS complex & fICI & $470(20-1095)$ & $1010(50-2040)$ & $1860(60-3260)$ \\
& AMBS & $790(670-1105)$ & $1060(690-2130)$ & $1990(800-3390)$ \\
Background noise amplitude $^{\text {b }}$ & & $850(660-1250)$ & $760(680-1330)$ & $810(750-1380)$ \\
Correlation coefficient $^{c}$ & & 0.245 & 0.645 & 0.805 \\
\hline
\end{tabular}

a Average peak-to-peak amplitudes of QRS complexes, given in fT, of fetal traces reconstructed with fICI and AMBS; for each fMCG data set averages were calculated on all 55 traces; minimum and maximum values are provided in parentheses.

b Average peak-to-peak amplitudes, given in fT, estimated on raw fMCG recordings.

c Average values of coupled correlation coefficients calculated between fetal traces reconstructed with fICI and AMBS; values refer to QRS complexes of all data sets comprised in the gestational period.

Finally, for each fetus the distance between inverse localizations $\left(x^{\prime}, y^{\prime}, z^{\prime}\right)$ and the atrioventricular node position $\left(x_{\mathrm{E}}, y_{\mathrm{E}}, z_{\mathrm{E}}\right)$ was compared with the tolerance limits assigned to each cardiac phase, and average figures were produced.

\section{Results}

\subsection{Reconstruction of 55 simultaneous fetal signals}

For each fMCG, 55 fetal cardiac signals were restored with fICI only when the quality of real-time recordings allowed retrieving a fetal trace from each cluster $C_{i}$. The number of effective fetal signal sets reconstructed from available fMCG with fICI and AMBS is given in table 1; average QRS waveforms and peak-to-peak amplitudes of signals obtained with the two methods were compared for each data set and for gestational periods, providing the figures in table 1.

An example of fetal trace reconstruction is given in figure 1, where two real-time fMCG recordings at 36 weeks are shown together with the corresponding fetal signals retrieved with fICI and AMBS; averaged beats are also provided for comparison. Figure 2 shows the distributions of averaged fetal beats obtained with fICI and AMBS for three patients at 24, 31 and 36 weeks' gestation.

\subsection{Fetal MCG mapping}

The performances of fICI and AMBS in reconstructing reliable field mapping were tested through inverse source localizations during P-QRS-T waves; tolerance limits and localization distances were calculated for each case; average figures are given for different gestational periods in table 2. The effectiveness of fICI in recovering as many reliable fetal signals as working acquisition channels (figure 2) entailed that detailed and dependable dipolar magnetic maps were available for all analyzed cases, while AMBS maps essentially reproduced noise distributions for early pregnancy, and not too definite dipolar maps also during late gestation. Three examples are given in figure 3: field maps were calculated for the same time instants from fICI and AMBS averaged fetal beats distributions (see figure 2). Localization details referring to those three cases are summarized in table 3 . 

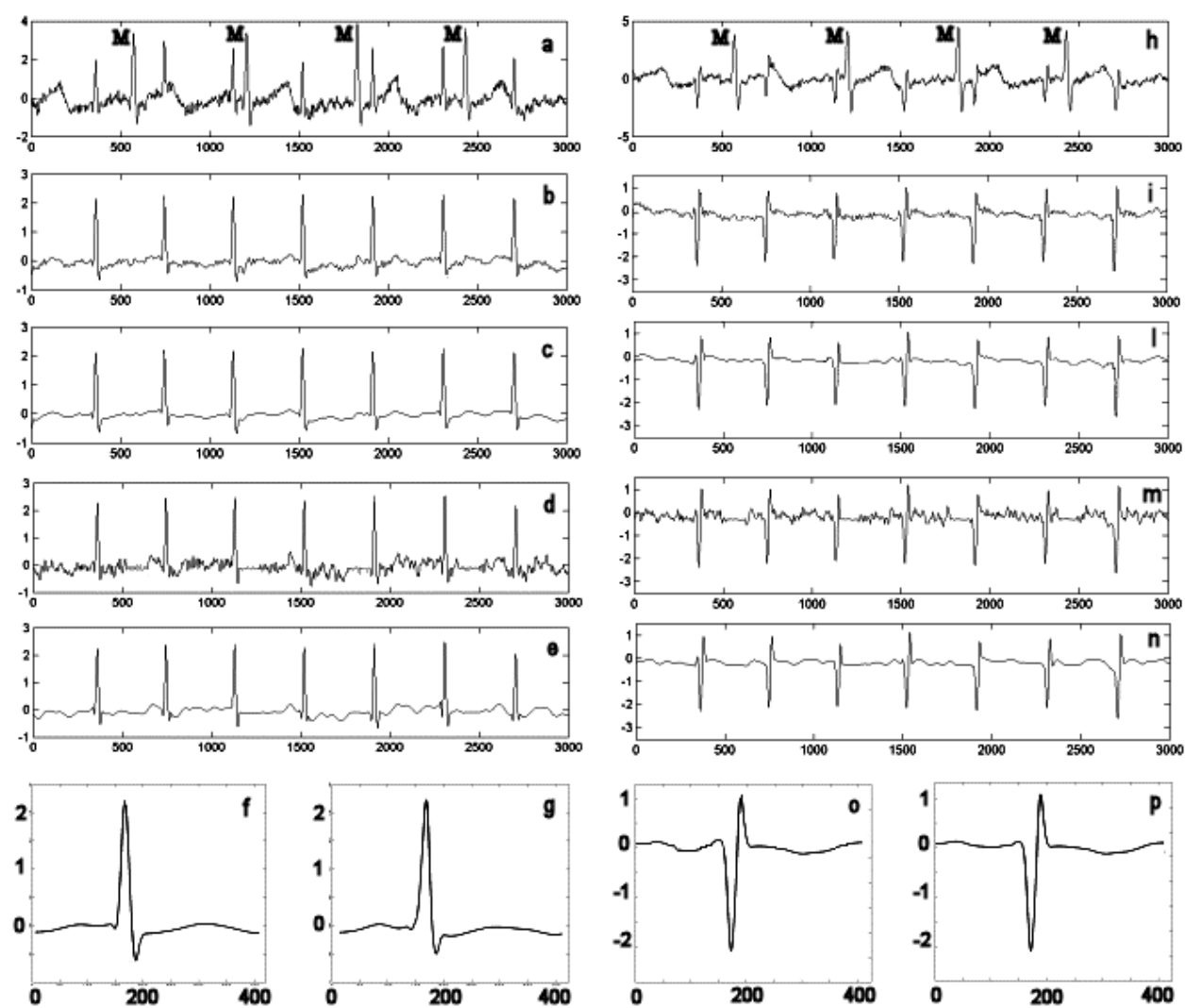

Figure 1. Real-time fMCG recordings (MCG channels no 0 and no 33) for a gravida at 36 weeks' gestation (a), (h); maternal QRS complexes are labeled with M, while the other peaks are fetal QRS complexes. Fetal signals reconstructed with fICI (b), (i) and smoothed (c), (1), and those retrieved with AMBS (d), (m) and smoothed (e), (n) are shown for comparison. Average fetal beats obtained from the signals displayed in (b), (i) and (d), (m) are shown in (f), (o) and (g), (p) respectively. Time is expressed in milliseconds and signals intensity is given in pT.

\section{Discussion}

FMCG is currently the most reliable method for the noninvasive surveillance of fetal cardiac function with the advance of gestation (Peters et al 2001). The fetal cardiac signals being much smaller than those from adult hearts, high resolution is required in time and space for a dependable fetal field mapping. This condition is satisfied when as many reliable fetal signals as available recordings are reconstructed from multichannel fMCG.

The weighted interpolation of fetal components obtained through FastICA iterations, performed on channel clusters tailored to optimize the distribution of retrieved fetal traces on the sensing plane, provided complete sets of 55 fetal traces with correct waveforms, intensities, polarities and relative positions: the better performance of fICI was particularly evident for early gestation. In fact, AMBS failed in restoring complete sets of consistent fetal traces when the intensity of fetal signals was lower than that of maternal signals or noise, while fICI, based on a technique able to separate noise as an independent component, which could therefore be disregarded, retrieved reliable fetal signals in the majority of cases, even when peak-to-peak amplitudes were lower than background noise. Moreover, the comparative 
fICI reconstructed signals

AMBS reconstructed signals

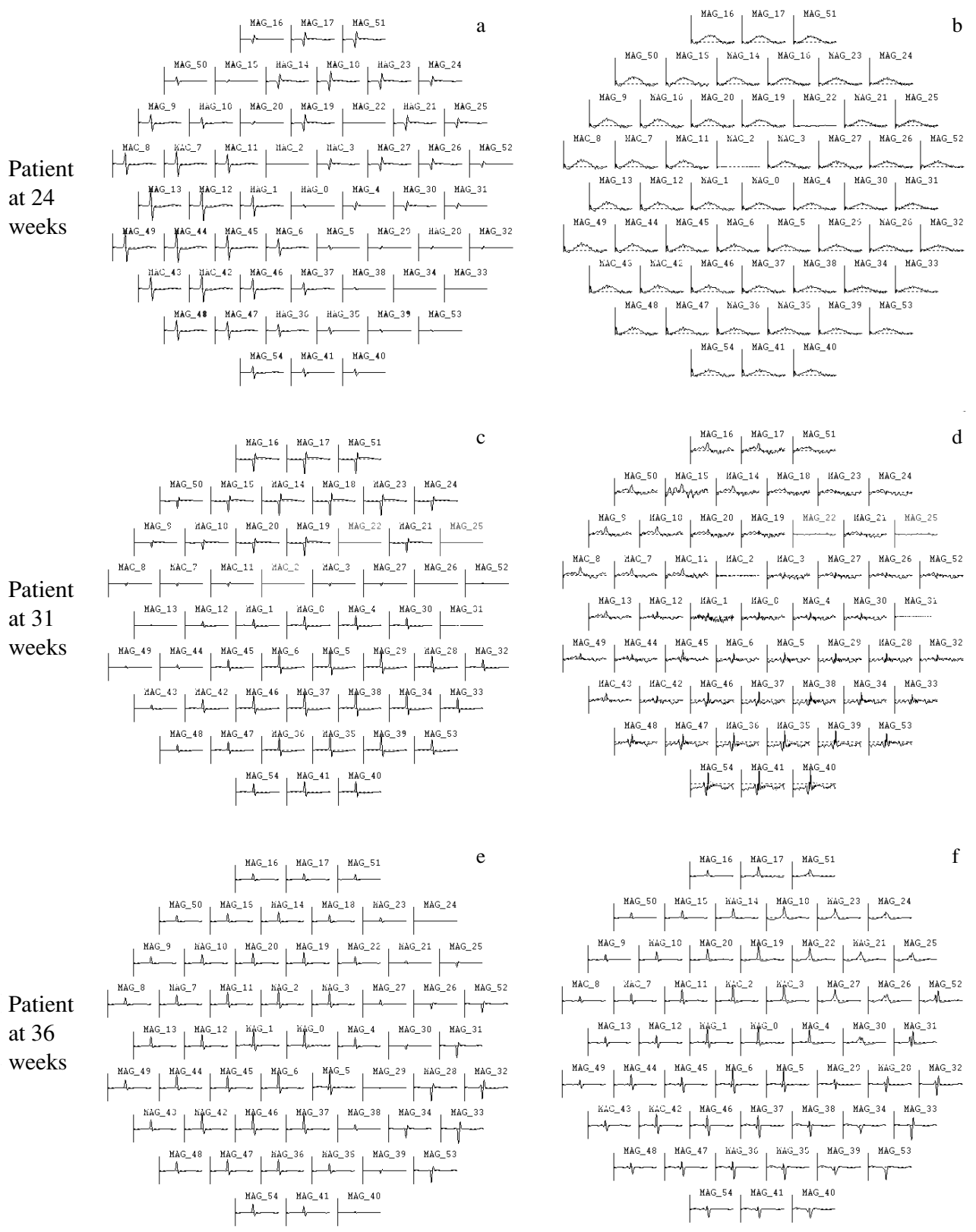

Figure 2. Examples of averaged fetal signals retrieved with fICI (a), (c), (e) and with AMBS (b), (d), (f); fetal traces are in the corresponding sensor positions. Data refer to three patients, respectively at 24 weeks (MCG channels no 2 and 22 were not working), at 31 weeks (MCG channels no 2, 22, 25 and 31 were not working) and at 36 weeks.

evaluation of QRS waveforms, quantified with average correlation coefficients, and peakto-peak intensities indicated that the performances of fICI and AMBS were similar for late 
Table 2. Comparison between the performances of fICI and AMBS in source localization.

\begin{tabular}{|c|c|c|c|c|c|}
\hline \multirow{2}{*}{\multicolumn{3}{|c|}{$\begin{array}{l}\text { Gestational period (weeks) } \\
\text { Number of fMCG with } \\
\text { ECHO data }\end{array}$}} & $22-27$ & $28-32$ & $33-37$ \\
\hline & & & 3 & 5 & 4 \\
\hline $\mathrm{P}$ wave ${ }^{\mathrm{a}}$ & $\begin{array}{l}\text { fICI } \\
\text { AMBS }\end{array}$ & $\begin{array}{l}\text { Tolerance limit }{ }^{\mathrm{c}} \\
\text { Localization distance }^{\mathrm{c}} \\
\text { Localization distance }^{\mathrm{c}}\end{array}$ & $\begin{array}{l}9.0 \pm 1.4 \\
10.5 \pm 2.1 \\
78^{b}\end{array}$ & $\begin{array}{l}10.5 \pm 1.5 \\
11.2 \pm 1.0 \\
90.0 \pm 18.5\end{array}$ & $\begin{array}{l}13.2 \pm 1.9 \\
10.7 \pm 1.5 \\
10.0 \pm 4.4\end{array}$ \\
\hline $\mathrm{R}$ wave ${ }^{\mathrm{a}}$ & $\begin{array}{l}\text { fICI } \\
\text { AMBS }\end{array}$ & $\begin{array}{l}\text { Tolerance limit } \\
\text { Localization distance }^{\mathrm{c}} \\
\text { Localization distance }^{\mathrm{c}}\end{array}$ & $\begin{array}{l}6.5 \pm 0.7 \\
7.5 \pm 0.7 \\
112^{\mathrm{b}}\end{array}$ & $\begin{array}{l}8.0 \pm 0.8 \\
7.2 \pm 1.0 \\
148.7 \pm 92.7\end{array}$ & $\begin{array}{l}10.2 \pm 1.0 \\
8.2 \pm 0.5 \\
8.8 \pm 0.9\end{array}$ \\
\hline T wave ${ }^{a}$ & $\begin{array}{l}\text { fICI } \\
\text { AMBS }\end{array}$ & $\begin{array}{l}\text { Tolerance limit } \\
\text { Localization distance }^{\mathrm{c}} \\
\text { Localization distance }^{\mathrm{c}}\end{array}$ & $\begin{array}{l}13.5 \pm 2.1 \\
5.5 \pm 3.5 \\
72^{\mathrm{b}}\end{array}$ & $\begin{array}{l}18.9 \pm 2.2 \\
9.5 \pm 2.5 \\
83.2 \pm 59.0\end{array}$ & $\begin{array}{l}20.9 \pm 2.8 \\
13.2 \pm 2.4 \\
16.8 \pm 3.4\end{array}$ \\
\hline
\end{tabular}

${ }^{\text {a }}$ For each patient the time instants used for fICI and AMBS localizations were the same.

${ }^{\mathrm{b}}$ Only 1 data set for AMBS.

c Tolerance limits and localization distances are given in $\mathrm{mm}$; values are expressed as average values \pm standard deviation; distances were calculated relative to the ECHO localization of the atrio-ventricular node and tolerance limits were estimated as a function of each fetal heart dimension.

gestation, but strongly diverging toward early pregnancy (table 1). Striking differences in signal morphology, particularly significant for $\mathrm{P}$ and $\mathrm{T}$ waves, could be appreciated with the decrease of gestational age, but also average beats and field mapping always indicated that fICI was superior to AMBS (figures 1 and 2).

The consistency of fICI field mapping was also demonstrated. A direct comparison between maps obtained with fICI and AMBS was possible only from the 27 th gestational week onward, either because complete sets of fetal signals retrieved with AMBS were unavailable, or because exceeding residual noise in AMBS averaged beats prevented any meaningful field map reconstruction. Three examples of fetal isofield mapping for P-QRS-T waves have been shown in figure 3. Although all maps have been calculated for the same time instants, distinct dipolar field distributions during the average cardiac cycle were obtained only from fetal signals retrieved with fICI, while dipolar, but less clearly defined, field maps were generated from AMBS traces only for late gestation because of poor traces quality. AMBS field maps calculated for earlier periods were generally very noisy.

The consistency of field mapping obtained with fICI and AMBS was proved verifying that the localizations of electrical sources fell inside an approximate cardiac volume reconstructed from morphological information on the fetal heart available from ultrasonography. The averaged figures shown in table 2 again indicate a marked superiority of fICI with respect to AMBS; this is also clear from the examples described with figures 2 and 3 and with table 3. In fact, only for the patient at 36 weeks AMBS field mapping allowed correct source localizations, while for the patient at 31 weeks all localizations fell far beyond the established tolerance limits, and no source position could be identified for the patient at 24 weeks because of inappropriate field maps.

On the other hand, localizations calculated using fICI mapping were always within the given boundaries for all waves, or very close to them, as in the case of the patient at 24 weeks, for which source position was within tolerance limits for the $\mathrm{T}$ wave, and $1 \mathrm{~mm}$ outside the approximate boundaries for the $\mathrm{P}$ wave and the beginning of $\mathrm{R}$ wave. Since the errors associated with localization, and disregarded in the present study, are generally around $6 \mathrm{~mm}$ (Comani et al 2003), the results attained for this patient can in any case be considered extremely good. 


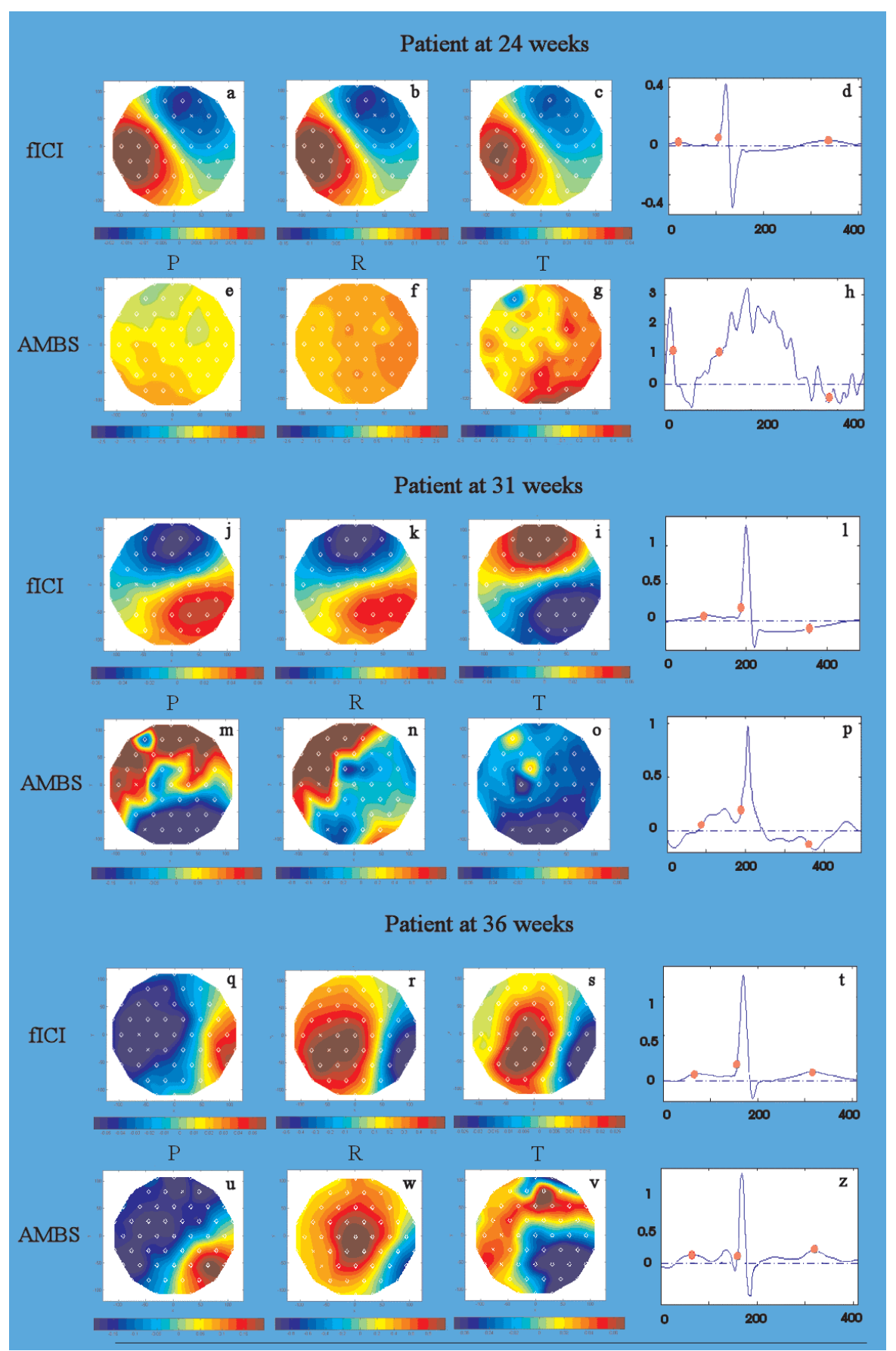

Figure 3. Fetal field mapping obtained from the averaged beats distributions shown in figure 2 . Each row consists of the isofield maps calculated for the time instants, during P-QRS-T waves, which are marked with a red dot on the averaged fetal beat displayed on the right. Field maps calculated with fetal traces restored with fICI are the figure groups $(a, b, c),(j, k, i)$ and (q, r, s), while those obtained from fetal signals retrieved with AMBS are the figure groups (e, f, g), $(\mathrm{m}, \mathrm{n}, \mathrm{o})$ and $(\mathrm{u}, \mathrm{w}, \mathrm{v})$. The averaged fetal beats displayed on the right correspond to MCG sensor position no 43 . 
Table 3. Equivalent cardiac source positions calculated through inverse localization using the isofield maps given in figure 3; all localizations are given as $(x, y, z)$ points in maternal coordinates and are expressed in $\mathrm{mm}$. Echocardiographic information on the fetal hearts is also provided.

\begin{tabular}{|c|c|c|c|c|c|}
\hline \multicolumn{3}{|l|}{ Gestational week } & $\begin{array}{l}\text { Patient no } 1 \\
24\end{array}$ & $\begin{array}{l}\text { Patient no } 2 \\
31\end{array}$ & $\begin{array}{l}\text { Patient no } 3 \\
36\end{array}$ \\
\hline \multicolumn{3}{|l|}{$\begin{array}{l}\text { Distance between maternal abdomen } \\
\text { and fetal heart center }(\mathrm{mm})\end{array}$} & 71.3 & 75.0 & 74.4 \\
\hline \multicolumn{3}{|l|}{$\begin{array}{l}\text { Fetal heart length from apex to } \\
\text { vertex }(\mathrm{mm})\end{array}$} & 23.7 & 41.3 & 44.7 \\
\hline \multicolumn{3}{|l|}{$\begin{array}{l}\text { Fetal heart width at interception } \\
\text { between atria and ventricula }(\mathrm{mm})\end{array}$} & 17.4 & 22.8 & 29.0 \\
\hline \multicolumn{3}{|l|}{ Fetal position } & Vertex LOA & Vertex LOA & Vertex ROA \\
\hline \multicolumn{3}{|c|}{ ECHO localization of atrio-ventricular node } & $(-17,33,-61)$ & $(38,16,-63)$ & $(38,-30,-57)$ \\
\hline \multirow{5}{*}{$\mathrm{P}$ wave $^{\mathrm{a}}$} & & Tolerance limit ${ }^{\mathrm{b}}$ & 8 & 14 & 15 \\
\hline & fICI & Localization & $(-20,38,-68)$ & $(26,12,-56)$ & $(43,-38,-62)$ \\
\hline & & distance $^{\mathrm{b}}$ & 9 & 14 & 11 \\
\hline & AMBS & Localization & NA & $(8,-30,-99)$ & $(39,-25,-60)$ \\
\hline & & distance $^{\mathrm{b}}$ & NA & 66 & 7 \\
\hline \multirow{5}{*}{ R wave wa $^{a}$} & & Tolerance limit ${ }^{\mathrm{b}}$ & 6 & 8 & 10 \\
\hline & fICI & Localization & $(-18,37,-67)$ & $(34,14,-57)$ & $(44,-34,-61)$ \\
\hline & & distance $^{\mathrm{b}}$ & 7 & 7 & 8 \\
\hline & AMBS & Localization & NA & $(-158,-98,-257)$ & $(46,-32,-57)$ \\
\hline & & distance $^{\mathrm{b}}$ & NA & 274 & 8 \\
\hline \multirow{5}{*}{ T wave ${ }^{\mathrm{a}}$} & & Tolerance limit ${ }^{\mathrm{b}}$ & 12 & 21 & 22 \\
\hline & fICI & Localization & $(-17,35,-62)$ & $(29,13,-66)$ & $(46,-29,-65)$ \\
\hline & & distance $^{\mathrm{b}}$ & 3 & 9 & 12 \\
\hline & AMBS & Localization & NA & $(76,-129,-126)$ & $(28,-25,-46)$ \\
\hline & & distance $^{\mathrm{b}}$ & NA & 162 & 16 \\
\hline
\end{tabular}

a Time instants used for fICI and AMBS localizations were the same for each patient.

${ }^{\mathrm{b}}$ Tolerance limits and distances are given in $\mathrm{mm}$; tolerance limits were calculated from the echocardiographic findings provided here; distances were calculated relative to the ECHO localization of the atrio-ventricular node and tolerance limits were calculated as a function of heart dimensions (see table 3) as explained in section 2.

NA $=$ Not available.

Finally, it is worth mentioning that the relative positions of the equivalent sources for $\mathrm{P}$ and $\mathrm{T}$ waves obtained with fICI mapping always fell in opposite half-spaces with respect to an ideal plane passing between atria and ventricula, as it should be. This fact is particularly remarkable for early gestation, given the extremely small dimensions of the fetal heart, and further confirms the reliability of fICI in retrieving sets of fetal signals suitable for accurate field mapping.

\section{Study limitations}

Available fMCG recordings refer to the second half of gestation; hence, fICI reliability could not be verified for earlier periods. Moreover, the validation of inverse localizations obtained from fICI and AMBS field mapping was possible only when ultrasonography and fMCG were performed one soon after the other, in order to avoid fetal displacements that could prevent any consistent comparison between inverse localization and ultrasonographic information. Although only 12 out of 60 available fMCG data sets fulfilled this condition, consistent results were provided. 


\section{Conclusions}

The obtained results, although preliminary, indicate that fICI is a dependable technique, especially valuable for early gestation, when classical techniques are ineffective. Although our outcomes need further verification on larger population and pregnancy period, it is our opinion that they have already demonstrated fICI effectiveness in reconstructing detailed sets of reliable fetal signals from multichannel fMCG. This was evident in particular when fetal traces retrieved with fICI and AMBS were comparable: equivalent source localizations attained with fICI field mapping were in fact always more consistent with ECHO information than those obtained with AMBS. Finally, since the authors have recently proved FastICA efficacy in retrieving reliable fetal signals also from unshielded fMCG recordings (personal communication), we are confident that fICI could be successful also for fMCG performed in a hospital setting, with a consequent invaluable clinical impact.

\section{References}

Brockmeier K et al 1997 Magnetocardiography and 32-lead potential mapping: repolarization in normal subjects during pharmacologically induced stress $J$. Cardiovasc. Electrophysiol. 8 615-26

Comani S et al 2001 Magnetic map analysis during ventricular repolarization to differentiate between normal subjects and patients affected by cardiac hypertrophy Phys. Med. 17 9-15

Comani S et al 2003 Magnetocardiographic functional imaging and integration with 3D MRI reconstruction of the heart: preliminary results for source localization during myocardium activation Phys. Med. 19 119-30

Comani S et al 2004a Concentric remodeling detection by magnetocardiography in patients with recent-onset arterial hypertension Pace 27 709-18

Comani S et al 2004b Detection of fetal arrhythmias by means of magnetocardiography: a case report Biomed. Tech . 48 156-8

Comani S et al 2004c Fetal intra-cardiac intervals for different gestational epochs as evaluated from fetal magnetocardiograms Biomed. Tech. 48 150-2

Comani S et al 2004d Time course reconstruction of fetal cardiac signals from fMCG: independent component analysis versus adaptive maternal beat subtraction Physiol. Meas. 25 1305-21

Comani S et al 2004e Independent component analysis: fetal signal reconstruction from magnetocardiographic recordings Comput. Methods Programs Biomed. 75 163-77

Della Penna S et al 2000 Biomagnetic systems for clinical use Phil. Mag. 80 937-48

Grimm B et al 2003 Influence of intrauterine growth restriction on cardiac time intervals evaluated by fetal magnetocardiography Early Hum. Dev. 74 1-11

Horigome $\mathrm{H}$ et al 2001 Detection of cardiac hypertrophy in the fetus by approximation of the current dipole using magnetocardiography Pediatr. Res. 50 242-5

Hosono T et al 2002 The coincidence of fetal magnetocardiography and direct electrocardiography in a case of fetal atrial flutter due to intracardiac tumor Fetal Diagn. Ther. 17 331-3

Hren R et al 1999 Value of magnetocardiographic QRST integral maps in the identification of patients at risk of ventricular arrhythmias Pace 22 1292-304

Hyvärinen A 1999 Fast and robust fixed-point algorithms for independent component analysis IEEE Trans. Neural Networks 10 626-34

Hyvärinen A and Oja E 2000 Independent component analysis: algorithms and applications Neural Networks 13 411-30

Kaler C et al 2002 Fetal magnetocardiography: development of the fetal cardiac time intervals Prenat. Diagn. 22 408-14

Kandori A et al 2001 A method for detecting myocardial abnormality by using a total current-vector calculated from ST-segment deviation of a magnetocardiogram signal Med. Biol. Eng. Comput. 39 21-8

Khaler C et al 2001 The application of fetal magnetocardiography (FMCG) to investigate fetal arrhytmias and congenital heart defects (CHD) Prenat. Diagn. 21 176-82

Kotini P A et al 2001 Fetal magnetocardiogram recordings and power spectra analysis in biomagnetic arrhythmic signals J. Obstet. Gynaecol. 21 368-72

Lewis M J 2003 Review of electromagnetic source investigations of the fetal heart Med. Eng. Phys. 25 801-10 
Lowery C L et al 2003 Noninvasive antepartum recording of fetal S-T segment with a newly developed 151-channel magnetic sensor system Am. J. Obstet. Gynecol. 188 1491-7

Mantini D et al 2004 Fetal tailoring of the independent component analysis to multi-channel fMCG recordings for an optimal reconstruction of the fetal cardiac signal Biomed. Tech. 48 186-8

Menendez T et al 2000 Prenatal diagnosis of QT prolongation by magnetocardiography Pace 23 1305-7

Menendez T et al 2001 Usefulness of magnetocardiography for the investigation of fetal arrhythmias Am. J. Cardiol. $88334-6$

Mitra S K and Kaiser F 1993 Handbook for Digital Signal Processing (New York: Wiley)

Muller H P et al 1999 Localization of a ventricular tachycardia-focus with multichannel magnetocardiography and three-dimensional current density reconstruction J. Med. Eng. Technol. 23 108-15

Pasquarelli A and Di Luzio S 1993 Instrumentation and methods for biomagnetic measurement Phys. Med. $9249-66$

Peters M et al 2001 Monitoring the fetal heart non-invasively: a review of methods J. Perinat. Med. 29 408-16

Siltanen P 1989 Magnetocardiography Comprehensive Electrocardiology ed P W Macfarlane and T D Veitch Lawrie (New York: Pergamon) pp 1405-38

St John Sutton M G et al 1984 Quantitative assessment of growth and function of the cardiac chambers in the normal human fetus: a prospective longitudinal echocardiographic study Circulation 69 645-54

Stinstra J et al 2002 Multicentre study of fetal cardiac time intervals using magnetocardiography Br. J. Obstet. Gynaecol. 109 1235-43

Tavarozzi I et al 2002a Magnetocardiography: current status and perspectives. Part I: Physical principles and instrumentation Ital. Heart J. 3 75-85

Tavarozzi I et al 2002b Magnetocardiography: current status and perspectives. Part II: Clinical applications Ital. Heart J. 3 151-65

Wakai R T, Lengle J M and Leuthold A C 2000a Transmission of electric and magnetic foetal cardiac signals in a case of ectopia cordis: the dominant role of the vernix caseosa Phys. Med. Biol. 45 1989-95

Wakai R T, Leuthold A C, Cripe L and Martin C B 2000b Assessment of fetal rhythm in complete congenital heart block by magnetocardiography Pace 23 1047-50

Wakai R T et al 2003 Magnetocardiographic rhythm patterns at initiation and termination of fetal supraventricular tachycardia Circulation 107 307-12

Weismuller P et al 1992 Magnetocardiographic non-invasive localization of accessory pathways in the WolffParkinson-White syndrome by a multichannel system Eur. Heart J. 13 616-22

Yang T N and Wang S D 1999 Robust algorithms for principal component analysis Phys. Rev. Lett. 20 927-33 